\title{
Adherence of Staphylococcus epidermidis to intraocular lenses
}

\author{
P G GRIFFITHS, ${ }^{1}$ T S J ELLIOT, ${ }^{2}$ AND L McTAGGART ${ }^{2}$
}

From the 'Department of Ophthalmology, Newcastle General Hospital, Westgate Road, Newcastle upon Tyne and the ${ }^{2}$ Queen Elizabeth Hospital, Queen Elizabeth Medical Centre, Edgbaston, Birmingham

SUMmARY We have demonstrated, with an in vitro model, that Staphylococcus epidermidis is able to colonise intraocular lenses. Adherent organisms were quantitated by light microscopy, scanning electron microscopy, and viable counting. Bacterial adherence was associated with production of a polysaccharide glycocalyx. Organisms which were attached to the lenses were resistant to apparently bactericidal concentrations of antibiotics, as determined by conventional testing. We speculate on the role of colonisation in the pathogenesis of endophthalmitis.

Staphylococcus epidermidis is now recognised as the commonest cause of endophthalmitis. ${ }^{1}$ This organism is normally considered to be of low pathogenicity, yet its frequency as a cause of endophthalmitis, as well as its occasional late presentation several months after surgery, requires explanation.

The commonest cause of infection of joint replacements and other prostheses, such as indwelling intravenous cannulae, is also Staph. epidermidis. This has been related to the organism's ability to adhere to plastic surfaces. ${ }^{2}$ There is evidence that this property is associated with the production of a polysaccharide glycocalyx,${ }^{3}$ which acts like a glue preventing mechanical removal. Furthermore 'slime' production confers resistance to bactericidal concentrations of antibiotics when the organisms are adherent to a smooth surface. ${ }^{45}$

Intraocular lenses may play a part in the pathogenesis of intraocular infections following cataract surgery. This proposition is supported by recent animal experiments which have shown that when Propionibacterium acnes was introduced into the anterior chamber at the conclusion of cataract surgery the presence of an intraocular lens made the eye more susceptible to infection. ${ }^{6}$ The resultant infection was also more intense and prolonged than intraocular infections in the absence of artificial lenses.

Bacterial adherence on to surfaces can be divided into two stages. The first stage involves reversible attraction due to electrostatic and Van der Waals

Correspondence to Mr P G Griffiths. forces, and hydrophobic bonding. The second stage involves slime production leading to irreversible adherence. Stage 1 adherence has been demonstrated by Vafidis et al. ${ }^{7}$ who wiped intraocular lenses round the wound site at the time of surgery and subsequently placed them in nutrient broth. In $26 \%$ of cases Staph. epidermidis grew in the broth. The lenses were not examined under the microscope after incubation in broth, so this figure is a measure of reversibly adherent organisms.

The present study was designed to determine whether or not stage 2 adherence to intraocular lenses occurs, resulting in firmly attached bacteria. An in-vitro model was developed and bacterial adherence was quantified by light microscopy, scanning electron microscopy, and estimation of viable counts.

\section{Materials and methods}

Two clinical isolates of Staph. epidermidis were used, namely, E004, a slime producer, and E001, a nonslime producer. An isolated colony of the test organism was taken from a nutrient agar plate and inoculated into $100 \mathrm{ml}$ of brain heart infusion broth (Sigma Laboratories), which was incubated at $37^{\circ} \mathrm{C}$ for 16 hours. $50 \mathrm{ml}$ of the culture was added to $100 \mathrm{ml}$ of fresh brain heart infusion broth, and the culture was used for the experiments described below.

Three different types of lens were used-closedloop anterior chamber lenses, and Rayner posterior chamber lenses style $850 \mathrm{~b}$ and 869 .

Lenses were suspended in the bacterial cultures by 
fine sterile wire threaded through the dialling holes or haptics. The lenses were then incubated at $37^{\circ} \mathrm{C}$ for variable periods of time as outlined below. The same type of lens was used for each experiment, so that the results were not biased by differential adherence to different lens surfaces. After all the experiments were completed a sample of the broth culture was subcultured on to nutrient agar to confirm purity.

Lenses were prepared for light microscopy by fixation in $3 \%$ glutaraldehyde in $0.1 \mathrm{M}$ sodium cacodylate buffer for two minutes, stained with methylene blue for five minutes, and washed by gentle agitation in three changes of distilled water in a $150 \mathrm{ml}$ beaker before being allowed to dry in air at $20^{\circ} \mathrm{C}$. The lenses were prepared for scanning electron microscopy by fixation in the $3 \%$ glutaraldehyde cacodylate buffer for two hours, followed by dehydration through ethyl alcohol of increasing concentrations $(50 \%, 75 \%, 90 \%, 95 \%$, and $100 \%)$ for 10 minutes. After critical freeze-point drying they were sputter coated with gold and examined under a Cambridge scanning electron microscope at an accelerating voltage of $7 \cdot 5-15 \mathrm{kV}$.
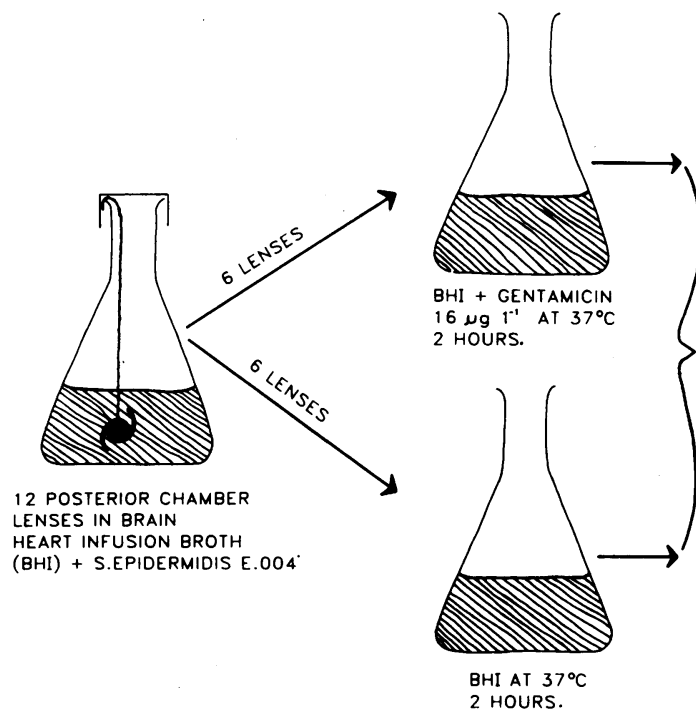

EACH LENS WASHED

IN FRESH BROTH

AT $37^{\circ} \mathrm{C}$ AND

PLACED IN TUBE

CONTAINING $1 \mathrm{mI}$ OF BROTH.

In a further experiment four lenses were incubated in a broth culture of Staph. epidermidis (E004) and four lenses in Staph. epidermidis (E001). One lens was
Fig. 1 Schematic illustration of experiment 1: determination of viable counts. 
removed from each culture at one, two, four, and 16 hours, washed in three changes of broth, and processed for scanning electron microscopy.

\section{EXPERIMENT 3 (Fig. 1)}

Twelve Rayner posterior chamber lenses were incubated for 90 minutes in a broth culture of Staph. epidermidis (E004). The lenses were removed and washed in fresh broth, six lenses were transferred to a broth containing gentamicin at a concentration of 16 $\mu \mathrm{g}^{-1}$ at $37^{\circ} \mathrm{C}$ for 90 minutes; the other six were placed in broth without gentamicin at $37^{\circ} \mathrm{C}$ for 90 minutes. Three lenses from each group were processed for light microscopy, the remaining lenses were washed in three changes of broth, and each lens was transferred to $1 \mathrm{ml}$ of broth, which was sonicated in a low-power cleaning sonicator for three bursts of 45 seconds each separated by 45 seconds (this regimen was found by prior experiments to remove all the adherent bacteria without affecting their viability). The specimens were vigorously mixed, and the broth containing the dislodged organisms was serially diluted, four times by a factor of 10 each time; $0.1 \mathrm{ml}$ of each dilution was pipetted on to separate nutrient agar plates and spread evenly across the surface of the agar with a sterile wire loop. The plates were incubated overnight at $37^{\circ} \mathrm{C}$, and the first plate with a small enough number of colonies to be counted were identified. On the assumption that each colony arises from one organism it is possible to calculate the number of organisms present in the original broth, and therefore on the surface of each lens, by multiplying by the appropriate power of 10 . The lenses which had been sonicated were stained for light microscopy to ensure that all adherent organisms had been removed.

Simultaneously $100 \mathrm{ml}$ of Staph. epidermidis culture was added to $200 \mathrm{ml}$ of brain heart infusion broth, and gentamicin was added to a concentration of $16 \mu \mathrm{g} \mathrm{l}^{-1}$. The mixture was incubated at $37^{\circ} \mathrm{C}$ for 90 minutes, when $5 \mathrm{ml}$ was removed and centrifuged at $2000 \mathrm{rpm}$ for five minutes. The precipitate was resuspended in $1 \mathrm{ml}$ of brain heart infusion broth and serially diluted for viable counting.

\section{Results}

EXPERIMENT 1

Slime producing and non-slime producing strains of Staph. epidermidis were able to adhere firmly to the surface of intraocular lenses (Table 1). For both strains this was further confirmed by demonstrating that there was no statistically significant difference in the number of attached organisms in lenses washed once, twice, or three times. Some of the adherent organisms were present in groups of two or three,
Table 1 Bacteria counts per high-power field after one, two, or three washes in broth: average of 10 high-power fields with standard deviation in parentheses

\begin{tabular}{|c|c|c|c|}
\hline & 1 Wash & 2 Washes & 3 Washes \\
\hline \multicolumn{4}{|c|}{ Posterior chamber lenses strain E004 } \\
\hline Lens 1 & $25(5 \cdot 0)$ & $32(9 \cdot 1)$ & $38(11 \cdot 0)$ \\
\hline Lens 2 & $34(7 \cdot 0)$ & $21(7 \cdot 0)$ & $33(6 \cdot 0)$ \\
\hline \multicolumn{4}{|c|}{ Anterior chamber lenses strain EOOI } \\
\hline & $19(4 \cdot 0)$ & $16(7 \cdot 0)$ & $17(6 \cdot 0)$ \\
\hline
\end{tabular}

suggesting that they were actively multiplying and colonising the surface of the lenses.

Only a few organisms were found to be adherent to the polypropylene haptics in comparison with the surface of the lens. However, the highly curved surface of the loops precluded accurate counting of attached organisms.

\section{EXPERIMENT 2}

It was not possible to take sufficient photographs for statistically significant counting, but it was readily apparent that the number of adherent organisms increased the longer the lens remained in broth culture. We have a subjective impression that more organisms were adherent to areas of the lens with surface irregularities (Fig. 2). After four hours of colonisation 'slime' surrounding the colonies was apparent (Fig. 3). The amount of slime increased over the next 12 hours (Fig. 4) and was also present, but in smaller amounts, on lenses colonised by strain E001 after 16 hours.

\section{EXPERIMENT 3}

There was an approximately 10 -fold reduction in the number of viable organisms attached to the lens

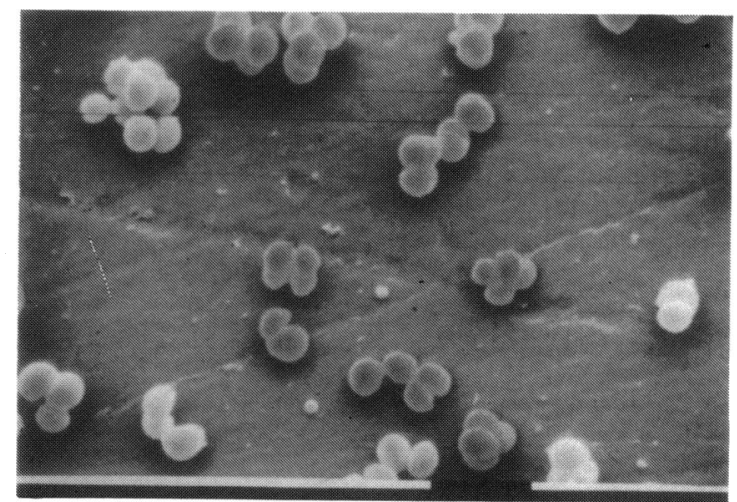

Fig. 2 Scanning electron microscope photography: Staph. epidermidis adherent to the surface of intraocular lens. The organisms appear to be preferentially colonising sites of surface irregularity. Black space bar $=2 \mu \mathrm{m}$. 


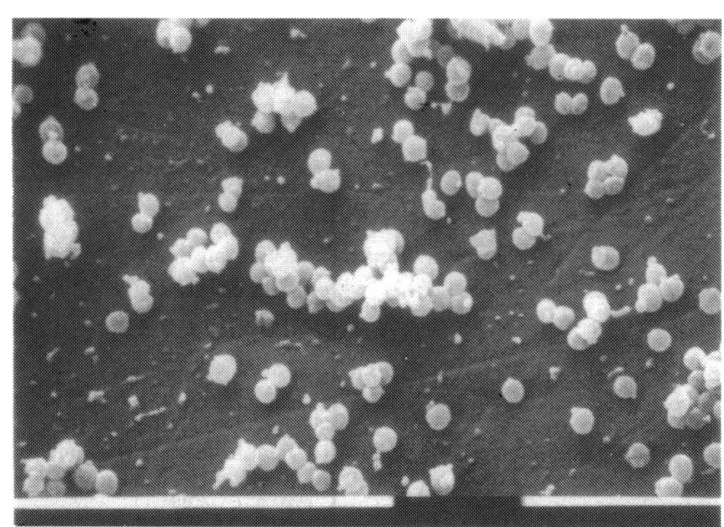

Fig. 3 Staph. epidermidis (E004) adherent to the surface of ' an intraocular lens after four hours' incubation. The fluffy material on the surface of some organisms represents the early stages of polysaccharide glycocalyx production. Black space bar $=4 \mu \mathrm{m}$.

following gentamicin treatment. The three lenses exposed to gentamicin for two hours had 6000,1000 , and 2000 organisms adhering, compared with 80000 , 40000 , and 70000 on the untreated lenses. When broth cultures were treated with the same concentration of gentamicin for a similar period of time no viable organisms were recovered.

The lenses processed for light microscopy still had large numbers of adherent organisms irrespective of treatment with gentamicin. The numbers in each high-power field were too great for accurate counting, but there was no subjective difference between the two groups. The lenses which were stained for light microscopy following sonication had only an occasional adherent micro-organism, demonstrating the efficacy of the sonication.

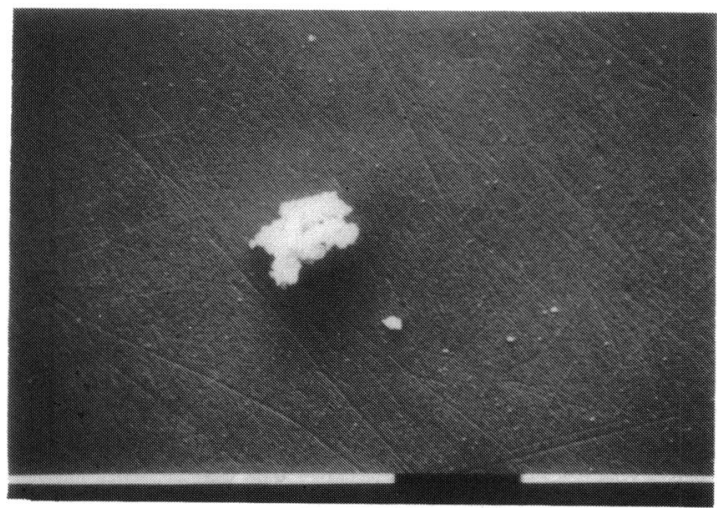

Fig. 4 Isolated colony of Staph. epidermidis (E004) adherent to the surface of an intraocular lens after 16 hours' incubation showing a more extensive polysaccharide coating. Black space bar $=10 \mu \mathrm{m}$.
Discussion

The results show that stage 2 adherence of Staph. epidermidis to intraocular lenses occurs, as evidenced by the resistance of organisms to removal by washing. Furthermore 'slime' production of Staph. epidermidis on the surface of an intraocular lens has been demonstrated for the first time. The appearance of a glycocalyx surrounding the organisms together with the increase in amount with time suggests that it has a major role in adherence to the lens, particularly stage 2 .

Surface finish of the lens may also be an important factor in adherence. More organisms appeared to be adherent to areas of irregular surface of the lens than of smooth surface. Previous workers have observed that surface defects provide a locus to which bacteria can initially adhere, ${ }^{8}$ and it seems likely that topographical defects of the lens encouraged colonisation.

The concentration of gentamicin found in the anterior chamber after the subconjunctival injection of $20 \mathrm{mg}$ of the antibiotic ${ }^{9}\left(16 \mu \mathrm{g} \mathrm{l}^{-1}\right)$ is sufficient to kill organisms in broth cultures, but it may not be sufficient to kill organisms adherent to the surface of a lens. However, it may be argued that in vivo organisms would not have had time to reach such an advanced state of adherence before being exposed to gentamicin given subconjunctivally at the conclusion of surgery, and that gentamicin is present in the anterior chamber for longer than two hours, though at a lower concentration. The mechanism by which adherence of bacteria to a synthetic surface confers resistance to antibiotics is unclear. However, it has been proposed that the slime coating prevents the antibiotics from gaining access to the organisms. ${ }^{10}$ Adherence of bacteria may also protect them from opsonising antibodies. ${ }^{\prime \prime}$

The role of intraocular lenses as a reservoir of bacteria in endophthalmitis has not been considered before. This study shows that Staph. epidermidis is capable of colonising lenses in vitro. Colonisation of lenses by Staph. epidermidis may be an important factor in the pathogenesis of endophthalmitis providing a surface to which bacteria can adhere where they are relatively resistant to antibiotics and possibly antibodies. The bacteria may subsequently cross the posterior capsule to multiply in the vitreous humour. It also offers an explanation for cases of localised endophthalmitis which have been described ${ }^{12}$ and cases of Staph. epidermidis endophthalmitis presenting late and recurring after apparently successful treatment with corticosteroids and antibiotics. ${ }^{13}$ If, like other prostheses, intraocular lenses play a part in causing localised infection, there may be new potential approaches for reducing the frequency of 
endophthalmitis. Stage 1 adherence might be reduced by avoiding contact with the conjunctiva during surgery and minimising transient electrostatic charge by washing the lens with balanced salt solution as soon as its container has been opened. Stage 2 adherence presents a more difficult problem, but attention to the surface finish of lenses may be important and materials other than polymethyl methacrylate such as silicone or poly-2-hydroxyethyl methacrylate (P-HEMA), which is less hydrophobic, may also assist in prevention of firm bacterial adherence. More detailed studies of the pathogenesis of endophthalmitis may provide further avenues for the prevention and treatment of this condition.

We thank Rayner Intraocular Lenses Ltd for providing the lenses used in this study, and Mr Angus Parker in the Department of Oral Anatomy at Newcastle University for his assistance with the electron micrographs.

\section{References}

1 Bohigan GM, Olk RJ. Factors associated with a poor visual result in endophthalmitis. Am J Ophthalmol 1986; 101: 332-4.

2 Gristina AG, Costerton JW, Leake ES, Kolkin J, Wright MJ. Bacterial colonization of biomaterials: clinical and laboratory studies. Orthopt Trans 1980; 4: 355.

3 Christenson GD, Simpson WA, Binso AL, Beachy EH.
Adherence of slime producing strains of Staphylococcus epidermidis to smooth surfaces. Infect Immun 1982; 37: 318-26.

4 Sheth NK, Franson TR, Sohnle PG. Influence of bacterial adherence to intravascular catheters on in-vitro susceptibility. Lancet 1985; ii: $1266-8$.

5 Dougherty SH, Simmons RL. Infection in bionic man: the pathobiology of infections in prosthetic devices-Part 1. Curr Probl Surg 1980; 19: 221-64.

6 Nobe JR, Finegold SM, Smith RE. Chronic anaerobic endophthalmitis in pseudophakic rabbit eyes. Invest Ophthalmol Vis Sci 1986; 27 (ARVO abstr suppl): 38.

7 Vafidis GC, Marsh RJ, Stacey AR. Bacterial contamination of intraocular lens surgery. Br J Ophthalmol 1984; 68: 520-3.

8 Elliot TSJ, Cheesebrough JS, Finch RG. Colonisation of intravenous cannulas. J Sterile Services Management 1984; 2: 21-2.

9 Litwack KD, Pettit T, Johnson BI. Penetration of gentamicin, administered intramuscularly and subconjunctivally into aqueous humour. Arch Ophthalmol 1969; 82: 687-93.

10 Elliot TSJ, D'Abrera VC, Dutton S. The effect of antibiotics on intravascular device bacterial colonisation. $\mathrm{J} \mathrm{Med} \mathrm{Microbiol}$ in press.

11 Baltimore RS, Mitchel M. Immunological investigations of mucoid strains of Pseudomonas aeruginosa: comparison of susceptibility by opsonic activity in mucoid and non-mucoid strains. J Infect Dis 1980; 141: 238-47.

12 Piest KL, Kincaid MC, Apple DJ, et al. Localized endophthalmitis: a newly recognized complication of cataract surgery and intraocular lens implantation. J Cataract Refract Surg 1987; 13: 481.

13 Valenton MJ, Brubaker RF, Allen HF. Staphylococcus epidermidis endophthalmitis. Arch Ophthalmol 1973; 89: 94-6.

Accepted for publication 14 July 1988. 